\title{
A!
}

This is an electronic reprint of the original article.

This reprint may differ from the original in pagination and typographic detail.

Pekola, Jukka; Holmqvist, Tommy; Meschke, Matthias

\section{Primary tunnel junction thermometry}

Published in:

Physical Review Letters

DOI:

10.1103/PhysRevLett.101.206801

Published: 14/11/2008

Document Version

Publisher's PDF, also known as Version of record

Please cite the original version:

Pekola, J., Holmqvist, T., \& Meschke, M. (2008). Primary tunnel junction thermometry. Physical Review Letters, 101(20), 1-4. [206801]. https://doi.org/10.1103/PhysRevLett.101.206801

This material is protected by copyright and other intellectual property rights, and duplication or sale of all or part of any of the repository collections is not permitted, except that material may be duplicated by you for your research use or educational purposes in electronic or print form. You must obtain permission for any other use. Electronic or print copies may not be offered, whether for sale or otherwise to anyone who is not an authorised user. 


\title{
Primary Tunnel Junction Thermometry
}

\author{
Jukka P. Pekola, Tommy Holmqvist, and Matthias Meschke \\ Low Temperature Laboratory, Helsinki University of Technology, P.O. Box 3500, 02015 TKK, Finland
}

(Received 1 August 2008; published 14 November 2008)

\begin{abstract}
We describe the concept and experimental demonstration of primary thermometry based on a fourprobe measurement of a single tunnel junction embedded within four arrays of junctions. We show that in this configuration random sample specific and environment-related errors can be avoided. This method relates temperature directly to Boltzmann constant, which will form the basis of the definition of temperature and realization of official temperature scales in the future.
\end{abstract}

DOI: 10.1103/PhysRevLett.101.206801

Temperature is a relatively poorly known quantity in modern metrology. It is well recognized that the way the international temperature scale is currently realized, in particular, towards low temperatures, needs to be seriously reconsidered. It is currently based largely on artifacts which should be replaced by methods relating to thermodynamic temperature via Boltzmann constant $k_{B}[1,2]$. Methods based on solid state tunnel junctions, Coulomb blockade thermometry (CBT) $[3,4]$ and shot noise thermometry (SNT) $[5,6]$, have both shown great promise as $k_{B}$-based thermometers for metrology. However, both of them fall short up to now, when it comes to absolute accuracy; for the range below $1 \mathrm{~K}$ accuracy of the order of $0.1 \%$ would be needed. In case of SNT, the limitations are mainly of practical nature, and can possibly be overcome by careful design. For CBT, an uncontrolled error source is of more fundamental concern: CBT involves a measurement of a series connection of nominally identical junctions. The inevitable spread in junction parameters leads, however, to an error, which can usually be made small, but which limits the accuracy, in particular, when the average junction size is small $[7,8]$. In this Letter we introduce and demonstrate a method, single-junction thermometry, SJT, where temperature can be inferred via a voltage measurement, based merely on the agreed value of the Boltzmann constant. SJT combines the advantages of basic CBT thermometry, but it avoids the parameter dispersion induced errors altogether. We show theoretically that the errors can then be efficiently suppressed, and demonstrate the operation in experiment.

In Coulomb blockade thermometry an array of tunnel junctions shows a drop in its differential conductance around zero bias voltage, because of the influence of single-electron charging effects. The ideal operation regime of a CB thermometer is determined by the ratio of the single-electron charging energy, $E_{C}=e^{2} / 2 C$, where $C$ is the (average) junction capacitance, and the thermal energy $k_{B} T$ at temperature $T$ such that $E_{C} \ll k_{B} T$. The measured conductance peak [see Fig. 1(a)] has two important characteristics, its full voltage width at half minimum, $V_{1 / 2}$, and its normalized (by asymptotic conductance at large voltages, $G_{T}$ ) depth $\Delta G / G_{T}$. Here the first one is given by
PACS numbers: 85.35.Gv, 06.20.-f, 07.20.Dt, 73.23.Hk

$5.44 k_{B} T / e$ per junction, and serves as the primary thermometer, provided the junctions in the sensor are mutually identical. The latter one is inversely proportional to $T$. SJT thermometry is based on the same principle as CBT but there the objective is to measure the conductance of a single tunnel junction, embedded in a four-probe configuration through lines consisting of arrays of tunnel junctions; see Fig. 1(b). In this topology, the advantageous protection from the influence of electromagnetic environment is achieved. At the same time, this configuration abolishes any requirement of a uniform structure, since only one junction is probed.

We separate the theoretical analysis into two parts. First we consider the case where the influence of the environment beyond the junction array can be neglected. We show that the measurement is perfect in this case even for a nonuniform structure. Then we use this result as the seed for analyzing the influence of the dissipative environment on the performance of the thermometer, and show that with sufficiently long junction arrays the accuracy can be maintained at the desired level.

The tunneling rate through a junction $i$ in forward $(+)$ or backward (-) direction with normal conductors in thermal equilibrium is given by [9]

$$
\Gamma_{0, i}\left(\delta F_{i}^{ \pm}\right)=\frac{1}{e^{2} R_{T, i}} \gamma\left(\delta F_{i}^{ \pm}\right),
$$

where $R_{T, i}$ is the junction resistance, $\gamma(x)=x /(1-$ $e^{-x / k_{B} T}$ ), and $\delta F_{i}^{ \pm}$is the change in electrostatic energy in
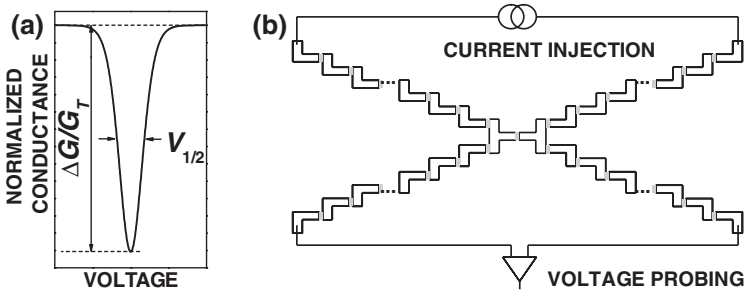

FIG. 1. The single-junction thermometer (SJT). (a) A typical conductance curve of a thermometer. (b) Schematics of the SJT. The white bounded areas are conductors, and the gray interconnects are tunnel junctions. 
tunneling. We may separate this energy change as $\delta F_{i}^{ \pm}=$ $\pm e V_{i}+\delta E_{\mathrm{ch}, i}^{ \pm}$, where $V_{i}$ is the (mean) voltage drop across junction $i$ and $\delta E_{\mathrm{ch}, i}^{+}$is the internal energy change associated with charging the capacitors of the array. In the present analysis we limit ourselves to the lowest order result in $E_{C} / k_{B} T$, which is what yields the basic results in thermometry. In this spirit, we then expand $\Gamma_{0, i}\left(\delta F_{i}^{ \pm}\right) \simeq$ $\Gamma_{0, i}\left( \pm e V_{i}\right)+\Gamma_{0, i}^{\prime}\left( \pm e V_{i}\right) \delta E_{\mathrm{ch}, i}^{ \pm}$. Analytic corrections for lower temperatures can be obtained readily by expanding up to higher orders, but they will not be considered here. The current $I_{i}$ through junction $i$ can be obtained as $I_{i}=$ $e \sum_{\{n\}} \sigma(\{n\})\left[\Gamma_{0, i}\left(\delta F_{i}^{+}\right)-\Gamma_{0, i}\left(\delta F_{i}^{-}\right)\right]$. Here, $\sigma(\{n\})$ is the occupation probability of the charge configuration $\{n\}$ on the islands within the array. With these premises, and by using identities $\gamma(x)-\gamma(-x)=x$ and $\gamma^{\prime}(x)+\gamma^{\prime}(-x)=$ 1 , we obtain

$$
\begin{aligned}
I_{i}= & \frac{1}{e R_{T, i}} \sum_{\{n\}} \sigma(\{n\})\left[e V_{i}+\left(\delta E_{\mathrm{ch}, i}^{+}+\delta E_{\mathrm{ch}, i}^{-}\right) \gamma^{\prime}\left(e V_{i}\right)\right. \\
& \left.-\delta E_{\mathrm{ch}, i}^{-}\right] .
\end{aligned}
$$

The internal charging energy for each charge configuration $\{n\}$ is given by $E_{\mathrm{ch}}=\frac{e^{2}}{2} \sum_{\{n\}}\left(\mathbf{C}^{-1}\right)_{i, j} n_{i} n_{j}$, where $\mathbf{C}^{-1}$ is the inverse capacitance matrix of the junction array. We have neglected the offset charges on the islands since in the high temperature regime the charge distribution is quasicontinuous, $\left\langle\delta n_{k}^{2}\right\rangle \gg 1$ [7]. Let us denote the islands surrounding the junction $i$ by $L$ and $R$. Then for the relevant processes only $n_{L}$ changes into $n_{L} \mp 1$ and $n_{R}$ to $n_{R} \pm 1$, whereas all the other charge numbers remain constant. There are two array connections to islands L and R in SJT. Evaluating $\delta E_{\mathrm{ch}, i}^{ \pm}$as the difference of $E_{\mathrm{ch}}$ for the charge configurations before and after the tunneling event, and making use of the properties $\sum_{\{n\}} \sigma(\{n\})=1$ and $\sum_{\{n\}} n_{k} \sigma(\{n\})=0$ for all $k$ because of the symmetry of $E_{\mathrm{ch}}$, we obtain the normalized conductance of junction $i,\left(G / G_{T}\right)_{i} \equiv R_{T, i} \frac{d I_{i}}{d V_{i}}$ as

$$
\left(\frac{G}{G_{T}}\right)_{i}=1-\frac{\delta_{i}}{k_{B} T} g\left(v_{i}\right),
$$

where $\delta_{i}=e^{2}\left[\left(\mathbf{C}^{-1}\right)_{L L}+\left(\mathbf{C}^{-1}\right)_{R R}-2\left(\mathbf{C}^{-1}\right)_{L R}\right], g(x)=$ $e^{x}\left[e^{x}(x-2)+x+2\right] /\left(e^{x}-1\right)^{3}$ and $v_{i}=e V_{i} / k_{B} T$. The result of Eq. (3) is in fact the basis of the standard CBT formula in linear arrays of junctions. Equation (3) tells that conductance of a single junction in SJT is accurate as a thermometer in any array of junctions: the magnitude of conductance suppression depends on the distribution of junction sizes via the capacitance matrix $\left(\delta_{i}\right)$, but the temperature can be determined unambiguously from, e.g., the half width of $g\left(v_{i}\right)$, if $V_{i}$ can be measured. Error-free measurement of $V_{i}$ is indeed possible in the configuration of Fig. 1(b). This happens since the voltage measurement via two arrays is typically performed using an amplifier with very large input impedance (in any case much larger than the resistance of the junction arrays). Then, essentially no current flows through these two ar- rays, and there is no voltage drop across them. Thus $V_{i}$ is indeed the voltage seen by the amplifier.

The analysis above applies to the case where the connection to the bias sources and signal amplifiers has zero impedance. In practice this is not the case, and the environment impedance introduces errors to Coulomb blockade thermometry which can be suppressed by using long arrays of junctions $[8,10]$. Similarly, one can realize the singlejunction measurement which avoids the errors by the embedding arrays. To analyze the remaining errors in this case quantitatively, we may write the tunneling rates $\Gamma_{i}^{ \pm}$instead of $\Gamma_{0, i}^{ \pm}$of Eq. (1) as

$$
\Gamma_{i}^{ \pm}=\frac{1}{e^{2} R_{T, i}} \int \gamma\left(E^{\prime}\right) P\left(\delta F_{i}^{ \pm}-E^{\prime}\right) d E^{\prime} .
$$

Here, $P(E)$ originates from the environment theory of single-electron tunneling [11], and it yields the probability (density) of electron to exchange energy $E$ when it tunnels: positive (negative) $E$ refers to energy emission (absorption) by electron. In the first analysis above we thus assumed $P(E)=\delta(E)$, i.e., the Dirac delta function. The same steps as in the ideal dissipationless environment above lead now to

$$
\begin{aligned}
\left(\frac{G}{G_{T}}\right)_{i}= & 1-\int\left[h\left(v_{i}+\frac{E}{k_{B} T}\right)-h\left(v_{i}-\frac{E}{k_{B} T}\right)\right] P(E) d E \\
& -\frac{\delta_{i}}{2 k_{B} T} \int\left[g\left(v_{i}+\frac{E}{k_{B} T}\right)\right. \\
& \left.+g\left(v_{i}-\frac{E}{k_{B} T}\right)\right] P(E) d E
\end{aligned}
$$

Here, $h(x)=e^{x}\left(e^{x}-x-1\right) /\left(e^{x}-1\right)^{2}$. For $P(E)=\delta(E)$, Eq. (5) reduces naturally to (3). Equation (5) yields an easy way to evaluate the influence of environment even in complex circuits.

In general $P(E)$ is obtained from the phase-phase correlation function $J(t)$ with $P(E)=\frac{1}{2 \pi \hbar} \int_{-\infty}^{\infty} d t \exp [J(t)+$ $\left.\frac{i}{\hbar} E t\right]$ [11]. Here we assume that the array is uniform and embedded in a resistive environment with resistance $R$. We are interested in conductance of one junction within an array. $J(t)$ can be written as

$$
\begin{aligned}
J(t)= & \pi \frac{R_{\mathrm{eq}}}{R_{K}}\left\{\left[\cot (B)\left(1-e^{-|\tau|}\right)\right.\right. \\
& \left.-\frac{|\tau|}{B}-2 \sum_{k=1}^{\infty} \frac{1-e^{-k \pi|\tau| / B}}{k \pi\left(1-(k \pi / B)^{2}\right)}\right] \\
& \left.-i\left[\operatorname{sgn}(\tau)\left(1-e^{-|\tau|}\right)\right]\right\} .
\end{aligned}
$$

Here $\tau=t /\left(R_{\mathrm{eq}} C_{\mathrm{eq}}\right), B=\hbar /\left(2 k_{B} T R_{\mathrm{eq}} C_{\mathrm{eq}}\right), R_{\mathrm{eq}}=R / N^{2}$ and $C_{\text {eq }}=N C$. For a linear array of $N$ junctions we have $\delta_{i}=\frac{N-1}{N} \frac{e^{2}}{C}$. The results for the corresponding SJT are identical to these upon replacing $N$ by $N^{\prime}+1$ in the expressions above, where $N^{\prime}$ is the number of junctions in each of the four surrounding arrays. Above we have assumed that the stray capacitances are small. 
Figure 2(a) shows the numerically calculated width of the conductance dip as normalized to that in delta-function environment for a junction in arrays with varying length and as a function of $R$. In general the peak is broadened; see Eq. (5). One needs to protect the junction by a long array, if accurate measurement of temperature using $V_{1 / 2}$ is to be obtained. For $N=2$, an error of about $10 \%$ in the range $100 \Omega \lessgtr R \lessgtr 500 \Omega$ is expected. The impedance of the environment at high frequencies is approximately $Z_{\text {env }}=\sqrt{\epsilon / \mu}$, determined by the permittivity $\epsilon$ and permeability $\mu$ of the medium. For vacuum its value is $\simeq 377 \Omega$, and for a circuit on silicon it is a few times smaller. Such increase of $V_{1 / 2}$ due to environment in short arrays is supported quantitatively by experiments, see, e.g., Ref. [7]. The depth of the conductance dip is shown in Fig. 2(b): it depends strongly on $R$ only in short arrays. We note the following. (i) Since the environment is never known precisely in the experiment, there is almost no way to correct theoretically for such errors: the only working strategy then is to suppress these errors precisely by embedding the measured junction in a long array. (ii) The effect of error suppression is essentially proportional to $N^{-2}$ (if $k_{B} T R C / \hbar<N$ ). Therefore, an array with $N \sim 50$ is in principle sufficient for measurements with $10^{-4} \mathrm{ab}$ solute accuracy, which would be sufficient for metrology in any conceivable temperature range of SJT. (iii) Embedding a junction in a very resistive environment [12] instead of a junction array is not the best strategy in thermometry either, which is indicated by the very slowly decaying tails of the error at large values of $R$. We show in Fig. 2(c) and 2(d) the width and depth, respectively, of a single-junction peak in a purely resistive environment. Although the width at large values of $R$ slowly approaches
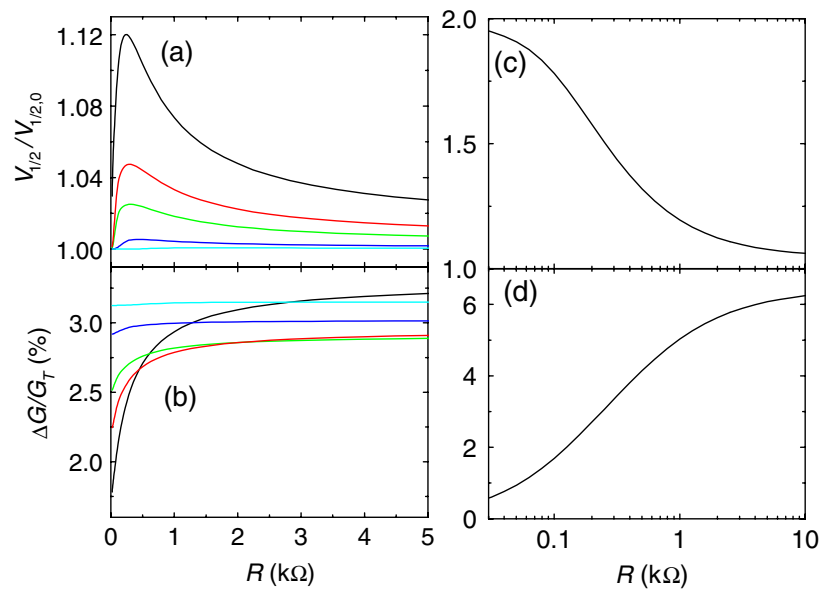

FIG. 2 (color online). The influence of environment on SJT thermometry. (a) $R$ dependence of the half width of the conductance dips for $N^{\prime}=1,2,3,7,15(N=2,3,4,8,16$ in linear arrays) from top to bottom. (b) The normalized depth of the conductance drop, with the same parameters as in (a). At low values of $R, N$ grows from bottom to up. (c),(d) The corresponding quantities on the logarithmic resistance scale for a single bare junction $\left(N^{\prime}=0, N=1\right)$. In these plots $E_{C} / k_{B} T=0.1$. unity, experimentally it is hard to fabricate resistive environments with $R \gg 10 \mathrm{k} \Omega$.

We discuss next the proof-of-the-concept experiments. Samples [see Fig. 3(a)] were fabricated by electron beam patterning and shadow angle evaporation. Both the bottom and the top electrodes are of aluminum, they are 40 and $45 \mathrm{~nm}$ thick, respectively. The bottom electrode was thermally oxidized at $100 \mathrm{mbar}$ for $10 \mathrm{~min}$ before deposition of the top electrode at an oblique angle. Two types of structures have been measured in this work. The single tunnel junction was connected either directly to the external leads or it was embedded within four arrays of $N^{\prime}=20$ junctions, respectively. The two types of structures were fabricated on the same chip in the same vacuum cycle. Nominally, the central junctions are identical in the two cases, and all the junctions are $0.6 \mu \mathrm{m}^{2}$ of area, yielding a junction resistance of $\simeq 6 \mathrm{k} \Omega$ (sample $A$ ) and $\simeq 4 \mathrm{k} \Omega$ (sample $B$ ).

The samples were measured in a dilution refrigerator with a $40 \mathrm{mK}$ base temperature. However, these structures were not suitable for very low temperature measurements: we observe strong self-heating due to weak electronphonon coupling in the present geometry near base temperature [13]. Therefore we present here data at temperatures at and above $150 \mathrm{mK}$. Conductance measurements for the SJT configuration and for the unprotected single junction are shown in Fig. 3(b) for sample $B$. Both the SJT $\left(N^{\prime}=20\right)$ and the bare junction $\left(N^{\prime}=0\right)$ data follow the environment calculation assuming $R=80 \Omega$, a value consistent with discussion above. One can verify the consistency with the model by comparing Figs. 2 and 3: the depth of SJT dip for $N^{\prime} \gg 1$ in Fig. 2(b) is about 2 times that in Fig. 3(d) for $R=80 \Omega$, whereas the normalized width in the former case is unity in Fig. 2(a), i.e., about one half of
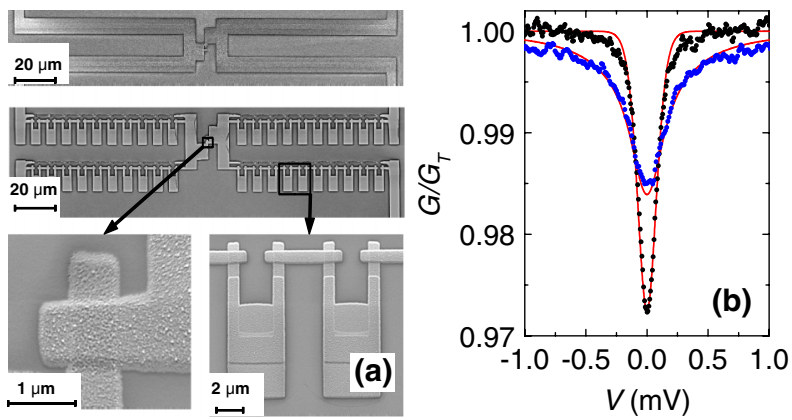

FIG. 3 (color online). Samples and comparison of the data on SJT and a bare single junction. (a) Electron micrographs of the reference structure with one junction connected to four leads (top), and the SJT structure with $N^{\prime}=20$ junctions in the leads (center). Zoom of the central junction and of a section of a junction array are shown at the bottom. (b) Measurement of the conductance of the bare single junction and the SJT in sample $B$ at $T \simeq 0.3 \mathrm{~K}$. The deeper and narrower drop in conductance corresponds to the SJT. The calculated conductance curves for the two samples based on the model described are shown by the solid lines assuming $R=80 \Omega$. 


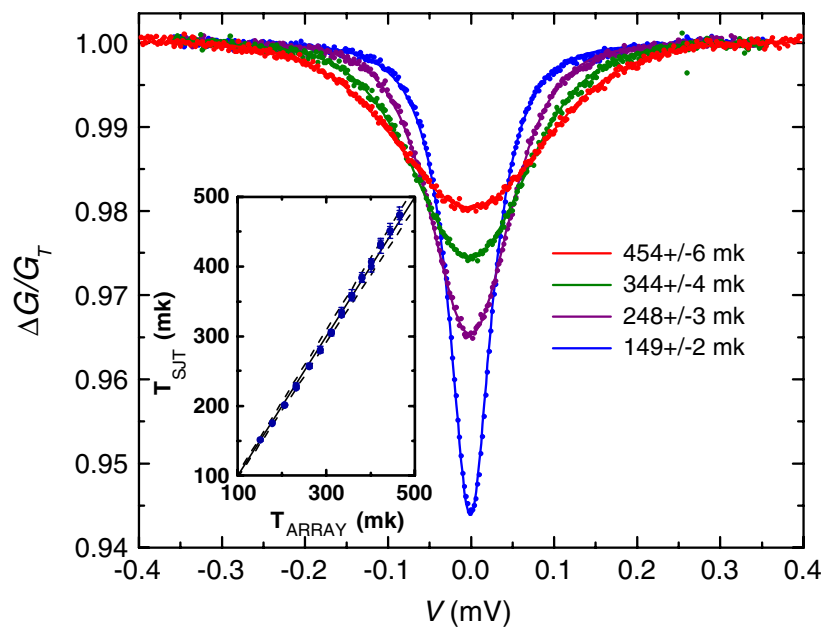

FIG. 4 (color online). Measurements on a SJT (sample A). Conductance dips at four temperatures are shown together with fits according to the model presented, including the selfheating correction [13]. The inset shows a comparison of the temperature deduced from SJT (vertical axis) against that obtained by an ordinary CBT measurement across one of the embedding arrays (horizontal axis). The solid line has unit slope, the error bars indicate the confidence interval of the fits, and the dashed lines the $\pm 3 \%$ uncertainty of the reference temperature scale.

that in Fig. 3(d) for a bare junction. The arrays can thus indeed be employed to efficiently protect the junctions against environment fluctuations; see also $[8,10,14]$.

Figure 4 shows four-probe conductance measurements of the SJT sample at a few temperatures together with fits according to the model presented. We included in the fits the influence of self-heating in the manner presented in Ref. [13], and this yielded perfect match to the peaks, see the lines on top of the data. Including the self-heating in the model influences the measured temperature by $0.8 \%$ at $450 \mathrm{mK}$ and by $6.6 \%$ at the lowest temperature shown. The extracted temperatures from the fits are shown in the inset of Fig. 4 against the reading of the CBT "reference thermometer", which was one of the $N^{\prime}=20$ junction arrays in the same sample. The dominating discrepancies between the two thermometers are due to finite errors in the fitting procedure in each case. The agreement between the two is good over the whole temperature range in Fig. 4. We expect that improved measurement uncertainty of $10^{-3}$ can be achieved by careful design of the measurement and setup.

In the theoretical analysis we focused on the high temperature and high junction resistance limit, and did not discuss errors due to, e.g., enhanced Coulomb effects at lower temperatures [8] and strong tunneling in low resistance junctions $[15,16]$. Yet these errors can be treated similarly to what has been done in standard Coulomb blockade thermometry, and their influence can be estimated and kept at a tolerable level by proper choice of junction sizes for each temperature and with suitable tunnel barrier parameters. One more (controllable) error to judge is the influence of the size of the islands between the junctions, $\ell$ : as long as it is smaller than the distance to "horizon", $\ell \ll \hbar c / k_{B} T$, the above lump element analysis is valid [17,18]. Here, $c=(\mu \epsilon)^{-1 / 2}$ is the signal propagation speed. The condition gets critical at particularly high temperatures, and extra care to place the array close to the junction has to be taken then. We want to add that the presented thermometry is not necessarily limited to the standard planar tunnel junction design, but may be applicable, e.g., in scanning probe or break junction geometries, since the junction parameters of the surrounding arrays need not be the same as those of the central junction. This might lead to the possibility of using tunable tunnel junctions in thermometry.

Summarizing, we propose an absolute single tunnel junction thermometer. We have demonstrated the concept in preliminary experiments. Our method may turn out to be valuable in future realization of the international temperature scale based on Boltzmann constant.

We thank Mikes (Centre for Metrology and Accreditation) and NanoSciERA project NanoFridge for financial support, and Dmitri Averin, Mikko Möttönen and Jost Engert for useful discussions.

[1] B. Fellmuth, Ch. Gaiser, and J. Fischer, Meas. Sci. Technol. 17, R145 (2006).

[2] G. Casa et al., Phys. Rev. Lett. 100, 200801 (2008).

[3] J.P. Pekola, K. P. Hirvi, J.P. Kauppinen, and M. A. Paalanen, Phys. Rev. Lett. 73, 2903 (1994).

[4] T. Bergsten, T. Claeson, and P. Delsing, J. Appl. Phys. 86, 3844 (1999).

[5] L. Spietz, K. W. Lehnert, I. Siddiqi, and R. J. Schoelkopf, Science 300, 1929 (2003).

[6] L. Spietz, R. J. Schoelkopf, and P. Pari, Appl. Phys. Lett. 89, 183123 (2006).

[7] K. P. Hirvi et al., Appl. Phys. Lett. 67, 2096 (1995).

[8] Sh. Farhangfar et al., J. Low Temp. Phys. 108, 191 (1997).

[9] D. V. Averin and K. K. Likharev, J. Low Temp. Phys. 62, 345 (1986)

[10] Sh. Farhangfar, A.J. Manninen, and J.P. Pekola, Europhys. Lett. 49, 237 (2000).

[11] G. L. Ingold and Yu. V. Nazarov, in Single Charge Tunneling, edited by H. Grabert and M.H. Devoret, NATO Advanced Study Institutes, Ser. B, Vol. 294 (Plenum Press, New York, 1992), p. 21.

[12] P. Joyez and D. Esteve, Phys. Rev. B 56, 1848 (1997).

[13] M. Meschke et al., J. Low Temp. Phys. 134, 1119 (2004).

[14] P. Delsing, K. K. Likharev, L. S. Kuzmin, and T. Claeson, Phys. Rev. Lett. 63, 1180 (1989).

[15] D. V. Averin and Yu. V. Nazarov, in Single Charge Tunneling, edited by H. Grabert and M.H. Devoret, NATO Advanced Study Institutes, Ser. B, Vol. 294 (Plenum Press, New York, 1992), p. 217.

[16] Sh. Farhangfar et al., Phys. Rev. B 63, 075309 (2001).

[17] J. P. Kauppinen and J. P. Pekola, Phys. Rev. Lett. 77, 3889 (1996).

[18] P. Wahlgren, P. Delsing, T. Claeson, and D. B. Haviland, Phys. Rev. B 57, 2375 (1998). 